\title{
Genome-wide identification and analysis of Catharanthus roseus RLK1-like kinases in Nicotiana benthamiana
}

Shaofei Rao ${ }^{1 \dagger}$, Xinyang $\mathrm{Wu}^{1,2 \dagger}{ }^{+}$Hongying Zheng ${ }^{1}$, Yuwen $\mathrm{Lu}^{1}$, Jiejun Peng ${ }^{1}$, Guanwei $\mathrm{Wu}^{1}$, Jianping Chen ${ }^{1}$ and Fei $\operatorname{Yan}^{1 *}$

\begin{abstract}
Background: The Catharanthus roseus RLK1-like kinase (CrRLK1L) is a subfamily of the RLK gene family, and members are sensors of cell wall integrity and regulators of cell polarity growth. Recent studies have also shown that members of this subfamily are involved in plant immunity. Nicotiana benthamiana is a model plant widely used in the study of plant-pathogen interactions. However, the members of the NbCrRLK1L subfamily and their response to pathogens have not been reported.
\end{abstract}

Results: In this study, a total of 31 CrRLK1L members were identified in the $N$. benthamiana genome, and these can be divided into 6 phylogenetic groups (I-VI). The members in each group have similar exon-intron structures and conserved motifs. NbCrRLK1Ls were predicted to be regulated by cis-acting elements such as STRE, TCA, ABRE, etc., and to be the target of transcription factors such as Dof and MYB. The expression profiles of the 16 selected NbCrRLK1Ls were determined by quantitative PCR. Most NbCrRLK1Ls were highly expressed in leaves but there were different and diverse expression patterns in other tissues. Inoculation with the bacterium Pseudomonas syringae or with Turnip mosaic virus significantly altered the transcript levels of the tested genes, suggesting that NbCrRLK1LS may be involved in the response to pathogens.

Conclusions: This study systematically identified the CrRLK1L members in N. benthamiana, and analyzed their tissue-specific expression and gene expression profiles in response to different pathogens and two pathogens associated molecular patterns (PAMPs). This research lays the foundation for exploring the function of NbCrRLK1Ls in plant-microbe interactions.

Keywords: Catharanthus roseus RLK1-like kinase, Nicotiana benthamiana, Genome-wide, Expression analysis

\footnotetext{
* Correspondence: yanfei@nbu.edu.cn

tShaofei Rao and Xinyang Wu contributed equally to this work.

${ }^{1}$ State Key Laboratory for Managing Biotic and Chemical Threats to the Quality and Safety of Agro-products, Key Laboratory of Biotechnology in

Plant Protection of Ministry of Agriculture and Zhejiang Province, Institute of

Plant Virology, Ningbo University, Ningbo 315211, China

Full list of author information is available at the end of the article
}

(c) The Author(s). 2021 Open Access This article is licensed under a Creative Commons Attribution 4.0 International License, which permits use, sharing, adaptation, distribution and reproduction in any medium or format, as long as you give appropriate credit to the original author(s) and the source, provide a link to the Creative Commons licence, and indicate if changes were made. The images or other third party material in this article are included in the article's Creative Commons licence, unless indicated otherwise in a credit line to the material. If material is not included in the article's Creative Commons licence and your intended use is not permitted by statutory regulation or exceeds the permitted use, you will need to obtain permission directly from the copyright holder. To view a copy of this licence, visit http://creativecommons.org/licenses/by/4.0/. The Creative Commons Public Domain Dedication waiver (http://creativecommons.org/publicdomain/zero/1.0/) applies to the data made available in this article, unless otherwise stated in a credit line to the data. 


\section{Background}

Receptor-like kinases belong to the RLK/Pelle gene family, which contains more than 600 members in the Arabidopsis genome and can be divided into 46 subfamilies. It plays an important role in plant growth, development and defense responses [1,2]. RLKs are composed of an extracellular domain (ECD), a transmembrane domain and an intracellular kinase domain [3]. The ECDs of plant RLKs are very diverse, and may include extensinlike domains, lectin-like domains, epidermal growth factor-like domains, lysine motifs, and leucine-rich repeat (LRR) domains [4]. RLK ECDs can undergo homologous or heterologous oligomerization to sense endogenous or exogenous ligands, including peptides, steroids, oligosaccharides, polysaccharides, and lipopolysaccharides, and transduce these signals to the inside of cells $[3,5,6]$.

Among the different clade members of plant RLKs, the Catharanthus roseus receptor-like kinase 1-like proteins (CrRLK1Ls) have received extensive attention in the past two decades because they regulate cell wall integrity [7]. Members of this subfamily are very conserved among all plants currently analyzed, including mosses and liverworts, indicating that the subfamily has an ancient origin [8]. CrRLK1L contains a carbohydrate binding domain (called malectin-like domain because it is similar to the malectin protein in animals), a transmembrane helix, and a C-terminal intracellular serine threonine kinase domain. The Arabidopsis genome contains 17 members, most of which have been functionally identified $[7,9]$. The widely studied member of CrRLK1L is the ubiquitously expressed FERONIA (FER). FER is necessary to recognize the pollen tube in female gametes. In synergids, FER is located at the filiform apparatus and is required to maintain the communication between the synergids and the pollen tube [10]. In addition, FER has growth-related functions in different types of cells in plants. For example, FER can regulate the apical growth of cells, including trichomes and root hairs [11]. FER also regulates the morphology of leaf epidermal cells by affecting the formation of lobes [12]. ANXUR1 (ANX1) and ANXUR2 (ANX2), which are pollen-specific and functionally redundant, are located on the plasma membrane at the tips of the pollen tube. Although they have the highest homology with FER, their function and tissue specificity are completely different $[13,14]$. ANX1 and ANX2 are necessary to maintain the integrity of the pollen tube during the apical polar growth process. The pollen tubes of an anx1 anx2 double mutant ruptured prematurely, leading to male sterility $[13,14]$. A recent study showed that the other two redundant members of the CrRLK1L subfamily expressed in pollen, BUDDHA'S PAPER SEAL1 (BUPS1) and BUPS2, are also necessary for the integrity of the pollen tube. BUPS1 and BUPS2 form complexes with ANX1 and ANX2, and both of them can bind to RALF4 and RALF19 expressed by pollen [15]. THESEUS1 (THE1) is reported to monitor the cell wall status. In the damaged cell wall environment, plants activate a THE1-dependent pathway to inhibit growth [16]. ERU is a positive regulator of cell growth and is very important for cytoplasmic $\mathrm{NH}_{4}^{+}$balance [17]. CURVY1 (CVY1), another CrRLK1L, plays an important role in the morphogenesis of trichome and tapetal cells, the transition from trophic state to reproductive state, and seed production [18]. CAP regulates the growth of calcium-dependent pollen tubes and participates in maintaining the composition of root hair cell walls during root tip growth $[16,19]$. The other four CrRLK1Ls, MEDOS1-4 are related to growth regulation in response to the presence of metal ions [20].

RAPID ALKALINIZATION FACTOR (RALF) peptides are reported to be ligands for some members of CrRLK1L [21, 22]. These peptides are widely distributed in terrestrial plants, and their activities are related to $\mathrm{pH}$ modulation and the production of reactive oxygen species (ROS) [22-24]. The RALF family has about 34 members in Arabidopsis, and they are differentially expressed in different plant tissues [25]. RALF1 treatment can inhibit the root and hypocotyl elongation of Arabidopsis seedlings [26, 27]. Recent studies have shown that RALF1 can directly bind to the extracellular domain of FER and induce the phosphorylation of proton pump AHA2, which is essential for the extracellular alkalinization and cell growth regulation induced by RALF1 [22]. RALF17, RALF23, RALF33, and RALF32 have also been shown to regulate FER. Similar to RALF1, RALF23, RALF33, and RALF32 can induce FERdependent seedling growth inhibition, and both RALF23 and RALF33 negatively regulate plant immunity [21]. Interestingly, RALF23 can inhibit the formation of FLAGELLIN-SENSING2 (FLS2)/ BRI1-ASSOCIATED RECEPTOR KINASE1 (BAK1) and ELONGATION FACTOR TU RECEPTOR (EFR)/ BAK1 complexes induced by pathogen-associated molecular patterns (PAMPs) flg22 and elf18 respectively through FER [21]. Recent structural and biochemical data indicate that LORELEI (LRE)-LIKE GLYCOSYLPHOSPHATIDYLINOSITOL (GPI)-ANCHORED PROTEIN 1 (LLG1) or LLG2 can directly bind to RALF23 to form a RALF23LLG1/2-FER heteropolymer complex, and other RALFs sharing a conserved N-terminal region with RALF23 may be perceived in a similar manner [28]. Ge et al. showed that RALF4 and RALF19 can bind to the extracellular domain of ANX1, ANX2, BUPS1 and BUPS2 with high affinity, indicating that these peptides are ligands of the ANX1/2-BUPS1/2 receptor complex [15].

Recent studies have shown that members of the CrRLK1L subfamily also have some roles in plant 
immunity. fer-2 and fer-4 mutants are less sensitive to ROS accumulation induced by efl 18 and flg22, and are more susceptible to Pseudomonas syringae pv. tomato DC3000 (Pst DC3000 COR ${ }^{-}$) infection. FER helps ligand-induced immune receptor FLS2, EFR and their co-receptor BAK1 to form a complex to initiate immune signals and positively regulate PAMP-trigered immunity [21]. It has been reported that FER can also inhibit JA and COR signals by phosphorylation and destabilization of MYC2, the main regulator of JA signalling, and positively regulates plant immunity. RALF23 stabilizes MYC2 and enhances JA signalling through FER to negatively regulate immunity [29]. ANX1 and ANX2 in Arabidopsis have been shown to negatively regulate the immune response mediated by Pattern Recognition Receptor (PRR) and Nod-Like Receptor (NLR). ANX1 binds constitutively with the bacterial flagellin receptor FLS2 and its co-receptor BAK1. After FLS2 senses flagellin, it can promote the binding of ANX1 and BAK1, so it will interfere with the formation of the FLS2-BAK1 complex to weaken the PRR signal [30]. In addition, ANX1 can form a complex with NLR protein RESISTANT TO PSEUDOMONAS SYRINGAE2 (RPS2) and RESISTANCE TO P. SYRINGAE PV MACULICOLA1 (RPM1), and ANX1 can promote the degradation of RPS2 to reduce RPS2-mediated cell death [30]. Another CrRLK1L member, LET1, was recently discovered to regulate the autoimmune pathway of mekk1-mkk1/2-mpk4 by forming a complex with the suppressor of $m k k 1 m k k 22$ (SUMM2) and MAP kinase kinase kinase 2 (MEKK2). The complex formed by MEKK2, LET1, and SUMM2 can fight against the ubiquitination and degradation of SUMM2 mediated by the F-box protein CPR1, thereby regulating the accumulation and activation of SUMM2 [31].

At present, members of CrRLK1L in Arabidopsis, Oryza sativa, Gossypium, Populus trichocarpa, Fragaria vesca and other plants have been identified [1, 32-36]. Nicotiana benthamiana is widely used to study plantpathogen interactions. However, the members of CrRLK1L in $N$. benthamiana have not been identified, and their response to different pathogens has not been reported. This study identified the members of CrRLK1L in N. benthamiana, and analyzed their tissue-specific expression patterns and gene expression profiles in response to different pathogens and two PAMPs. This research lays the foundation for further research on the function of $\mathrm{NbCrRLK1L}$ in plant-microbe interactions.

\section{Results}

Genome-wide identification and naming of $\mathrm{NbCrRLK1L}$ members

CrRLK1L contains an extracellular malectin-like domain, a transmembrane helix, and an intracellular kinase domain. We identified possible CrRLK1L members in
Nicotiana benthamiana based on these criteria. The amino acid sequences of the 17 identified Arabidopsis CrRLK1L members were downloaded from TAIR (http://www.arabidopsis.org/), and the $N$. benthamiana genome sequence was downloaded from Sol Genomics Network (https://solgenomics.net/). After two rounds of BLASTP, $31 \mathrm{NbCrRLK1Ls}$ were identified (Additional file 1: Table S1). These members were named NbCrRLK1L1 to NbCrRLK1L31 according to their chromosome locations (Table 1). The vast majority of members contain about 800 amino acids. The largest contains 954 aa, and the smallest only 439 aa. Their molecular weights are between 50 and $106 \mathrm{kDa}$, and their theoretical isoelectric points range between 5.3 and 6.54 (Table 1).

\section{Phylogenetic analysis}

In order to better understand the evolutionary relationship of the CrRLK1L subfamily genes in Arabidopsis and $N$. benthamiana, the amino acid sequences of 17 Arabidopsis and $31 N$. benthamiana members were used to construct a phylogenetic tree. Based on the classification of CrRLK1Ls in rice [32], the $31 \mathrm{NbCrRLK1L}$ genes could be divided into 6 groups. As shown in Fig. 1, there are 11 members in group I (NbCrRLK1L4, $-23,-22$, $1,-24,-14,-16,-9,-10,-18,-28), 5$ members in group II (NbCrRLK1L11, - 12, - 20, - 13, - 29), 3 members in group III (NbCrRLK1L15, - 27, - 17), 2 members in group IV (NbCrRLK1L5, - 7), 8 members in group V (NbCrRLK1L8, $-19,-2,-25,-21,-26,-6,-31$ ), and 2 members in group VI (NbCrRLK1L3, - 30). At least one CrRLK1L from Arabidopsis thaliana was included in each group except VI. The first and fifth groups have the most members, indicating that members in different groups have experienced different evolutionary events.

\section{Analysis of NbCrRLK1L conserved motifs, gene structure and domains}

To further understand the evolution of CrRLK1L members, we compared conserved motifs, functional domains and the exon-intron organization of NbCrRLK1L members. An online MEME analysis was used to identify motifs among the $31 \mathrm{NbCrRLK} 1 \mathrm{~L}$ members, and a total of 10 conserved motifs were predicted (Additional file 2: Table S2). The distribution of NbCrRLK1L motifs is relatively similar (Fig. 2a and b). Most members contain 9-10 motifs, except for NbCrRLK1L6, which has only five motifs. NbCrRLK1L27 contains 8 motifs; $\mathrm{NbCrRLK1L3}$ and NbCrRLK1L30 contain 7 and 6 motifs respectively (Fig. 2b). Most members of $\mathrm{NbCrRLK1L}$ have no introns (Fig. 2c), consistent with the findings from other plants including Arabidopsis, rice, poplar, strawberry, cotton and legumes [24-28]. Five members $(\mathrm{NbCrRLK1L} 18,-28,-24,-8,-31)$ have a single 
Table 1 Detailed information of the 31 predicted CrRLK1Ls proteins in N. benthamiana

\begin{tabular}{|c|c|c|c|c|c|c|c|c|}
\hline Gene Symbol & Gene Locus & Gene Position & Chromosome Location & Strand & CDS (bp) & $\begin{array}{l}\text { Protein } \\
\text { Length (aa) }\end{array}$ & $\begin{array}{l}\text { Molecular } \\
\text { weight (kDa) }\end{array}$ & $\begin{array}{l}\text { Theoretical } \\
\text { PI }\end{array}$ \\
\hline NbCrRLKIL1 & Niben101Scf00063g05010.1 & Niben101Scf00063 & Niben101Scf00063:516491,519040 & - & 2547 & 849 & 94.20 & 5.99 \\
\hline NbCrRLK1L2 & Niben101Scf00687g08007.1 & Niben101Scf00687 & Niben101Scf00687:834396,837032 & - & 2634 & 878 & 97.23 & 5.78 \\
\hline NbCrRLK $1 L 3$ & Niben101Scf01018g02004.1 & Niben101Scf01018 & Niben101Scf01018:258892,264285 & + & 2712 & 904 & 100.87 & 5.71 \\
\hline$N b C r R L K 1 L 4$ & Niben101Scf01025g13013.1 & Niben101Scf01025 & Niben101Scf01025:1432073,1434643 & - & 2568 & 856 & 94.68 & 5.27 \\
\hline NbCrRLK K1L5 & Niben101Scf01061g08039.1 & Niben101Scf01061 & Niben101Scf01061:950012,952540 & - & 2526 & 842 & 93.45 & 5.89 \\
\hline NbCrRLK1L6 & Niben101Scf01075g00017.1 & Niben101Scf01075 & Niben101Scf01075:37046,47016 & - & 1317 & 439 & 50.15 & 5.75 \\
\hline NbCrRLKIL7 & Niben101Scf01395g00019.1 & Niben101Scf01395 & Niben101Scf01395:28554,42854 & + & 2862 & 954 & 106.06 & 5.30 \\
\hline NbCrRLK1L8 & Niben101Scf01934g04017.1 & Niben101Scf01934 & Niben101Scf01934:510898,513583 & - & 2388 & 796 & 87.87 & 6.30 \\
\hline NbCrRLK1L9 & Niben101Scf01976g00011.1 & Niben101Scf01976 & Niben101Scf01976:181105,183618 & - & 2511 & 837 & 91.68 & 5.42 \\
\hline NbCrRLK1L10 & Niben101Scf01992g01013.1 & Niben101Scf01992 & Niben101Scf01992:157667,160823 & - & 2652 & 884 & 96.92 & 5.33 \\
\hline NbCrRLK1L11 & Niben101Scf02441g04008.1 & Niben101Scf02441 & Niben101Scf02441:525793,528408 & - & 2613 & 871 & 96.68 & 6.03 \\
\hline NbCrRLK1L12 & Niben101Scf02449g02004.1 & Niben101Scf02449 & Niben101Scf02449:261360,277268 & - & 2124 & 708 & 78.65 & 5.88 \\
\hline NbCrRLK1L13 & Niben101Scf02639g04006.1 & Niben101Scf02639 & Niben101Scf02639:387053,389425 & + & 2370 & 790 & 88.96 & 5.58 \\
\hline NbCrRLK1L14 & Niben1015cf02712g00006.1 & Niben101Scf02712 & Niben101Scf02712:14349,16844 & - & 2493 & 831 & 91.49 & 5.37 \\
\hline NbCrRLK1L15 & Niben101Scf02793g03001.1 & Niben101Scf02793 & Niben101Scf02793:351588,355656 & - & 2190 & 730 & 81.73 & 6.86 \\
\hline NbCrRLK1L16 & Niben101Scf03407g02009.1 & Niben101Scf03407 & Niben101Scf03407:214538,217054 & - & 2514 & 838 & 92.55 & 5.77 \\
\hline NbCrRLK1L17 & Niben101Scf03747g00006.1 & Niben101Scf03747 & Niben101Scf03747:58947,61529 & - & 2580 & 860 & 96.20 & 5.53 \\
\hline NbCrRLK1L18 & Niben101Scf03794g01015.1 & Niben101Scf03794 & Niben101Scf03794:244820,248030 & + & 2301 & 767 & 85.21 & 6.51 \\
\hline NbCrRLK1L19 & Niben101Scf03905g01001.1 & Niben101Scf03905 & Niben101Scf03905:172169,174751 & + & 2580 & 860 & 95.29 & 6.69 \\
\hline NbCrRLK1L20 & Niben101Scf03939g00003.1 & Niben101Scf03939 & Niben101Scf03939:45922,48414 & + & 2490 & 830 & 91.39 & 5.94 \\
\hline NbCrRLK1L21 & Niben101Scf04123g01031.1 & Niben101Scf04123 & Niben101Scf04123:265055,267721 & - & 2664 & 888 & 96.81 & 6.09 \\
\hline NbCrRLK1L22 & Niben101Scf04861g00003.1 & Niben101Scf04861 & Niben101Scf04861:29496,32135 & + & 2637 & 879 & 96.93 & 5.77 \\
\hline NbCrRLK1L23 & Niben101Scf05300g01006.1 & Niben101Scf05300 & Niben101Scf05300:186398,188962 & - & 2562 & 854 & 94.45 & 5.40 \\
\hline NbCrRLK1L24 & Niben101Scf05928g00006.1 & Niben101Scf05928 & Niben101Scf05928:421 10,44983 & + & 2559 & 853 & 94.72 & 5.67 \\
\hline NbCrRLK1L25 & Niben101Scf07442g01027.1 & Niben101Scf07442 & Niben101Scf07442:255193,257823 & - & 2628 & 876 & 97.05 & 6.15 \\
\hline NbCrRLK1L26 & Niben101Scf07619g00006.1 & Niben101Scf07619 & Niben101Scf07619:26955,29621 & - & 2664 & 888 & 96.62 & 6.02 \\
\hline NbCrRLK1L27 & Niben101Scf08500g01001.1 & Niben101Scf08500 & Niben101Scf08500:153954,156035 & + & 2079 & 693 & 77.39 & 8.91 \\
\hline NbCrRLK1L28 & Niben101Scf10015g05008.1 & Niben101Scf10015 & Niben101Scf10015:550054,552437 & - & 1947 & 649 & 72.03 & 6.33 \\
\hline NbCrRLK1L29 & Niben101Scf10015g10004.1 & Niben101Scf10015 & Niben101Scf10015:1 109772,1112147 & + & 2373 & 791 & 88.78 & 5.71 \\
\hline NbCrRLK1L30 & Niben101Scf25547g00009.1 & Niben101Scf25547 & Niben101Scf25547:10845,21227 & - & 2697 & 899 & 100.02 & 5.30 \\
\hline NbCrRLK1L31 & Niben101Scf25768g00010.1 & Niben101Scf25768 & Niben101Scf25768:9117,12488 & - & 2640 & 880 & 100.02 & 6.54 \\
\hline
\end{tabular}

intron, while $N b C r R L K 1 L 3$ and $N b C r R L K 1 L 30$, have multiple introns. All 31 members have the conserved malectin-like domain and the protein kinase domain (Fig. 2c).

\section{Prediction of promoter cis elements and transcription factors}

The cis-acting elements of the $N b C r R L K 1 L$ promoter region were analyzed, and a total of 4154 cis-acting elements of 83 types were predicted (Additional file 3: Table S3). These cis-acting elements are related to environmental stress, hormonal response, development, light response, site binding, promoters, and other functions. The most numerous elements are promoter-related elements, with $43-130$ in each $N b C r R L K 1 L$ (Fig. 3a). A total of 229 elements related to environmental stress were predicted in 10 categories, the largest numbers of which were STRE, TCA and ARE elements (Fig. 3b). A total of 352 hormone-related components were predicted in 13 categories, mainly related to ABA, JA, GA, and auxin (Fig. 3c).

In order to show the regulatory network of $N b C r R L K 1 L$ in cells more comprehensively, we predicted the possible transcription factors of $\mathrm{NbCrRLK1L}$. No corresponding transcription factors were predicted for $N b C r R L K 1 L 15$ and NbCrRLK1L27, but among the others a total of 23 types of transcription factors were predicted to regulate the expression of this subfamily (Fig. 4 and Additional file 4: Table S4). Among them, Dof, MIKE-MADS, TCP and MYB transcription factors were the most abundant. The different genes were regulated by an average of 7 transcription factors, with 


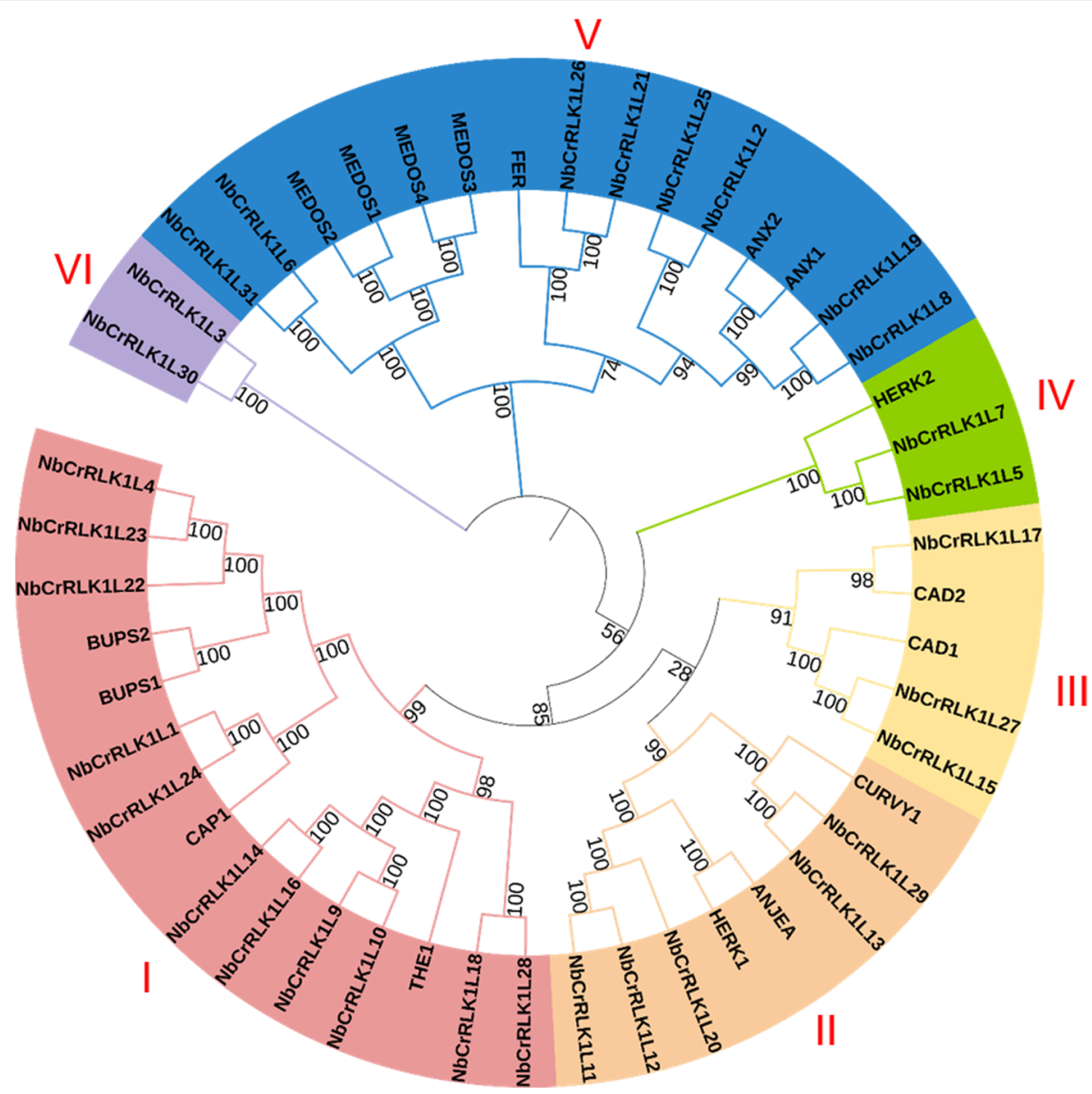

Fig. 1 Phylogenetic tree of malectin-like domain-containing proteins in N. benthamiana and A. thaliana. The phylogenetic tree was constructed using CrRLK1L amino acid sequences by the neighbor-joining method in MEGA X with 1000 bootstrap replicates. The phylogenetic tree was divided into six groups, which are shown in different colors, and identified by red Roman numerals

NbCrRLK1L4/13/18/21/22/23/26/28/29 regulated by 10 transcription factors, while $N b C r R L K 1 L 1$ and $\mathrm{NbCrRLK1L24}$ can be regulated by only two (Fig. 4).

\section{NbCrRLK1L tissue-specific expression}

The expression levels of all NbCrRLK1Ls were determined in each of the five tissue types (roots, stems, young leaves, mature leaves, flowers), and we here present the results of 16 members that were expressed in all five tissues and which represent each of the phylogenetic groups. Except for NbCrRLK1L8, NbCrRLK1L17 and $N b C r R L K 1 L 31$, the genes were expressed at a higher level in young leaves than in mature ones (Fig. 5 and Additional file 5: Figure S1). NbCrRLK1L11, 15, 16, 17, $21,26,28,29,31$ are expressed at high levels in leaves, implying that these members may be involved in responding to external stimuli (Fig. 5 and Additional file 5: Figure S1). $N b C r R L K 1 L 8$ is expressed at high levels in flowers, $N b C r R L K 1 L 30$ is expressed at high levels in roots and $N b C r R L K 1 L 3,20,21$ are highly expressed in the stem (Fig. 5 and Additional file 5: Figure S1) showing differences among the genes in their tissue-specific expression patterns.

\section{Induction of $N b C r R L K 1 L$ expression by pathogens and PAMPs}

To explore whether NbCrRLK1Ls are involved in the response to pathogens, we inoculated Pseudomonas syringae pv tomato strain DC3000 (Pst DC3000), three viruses (TuMV, PMMoV, PVX) and two PAMPs onto $N$. benthamiana leaves. Two days after Pst DC300 infection, 5 days after virus inoculation and $3 \mathrm{~h}$ after PAMPs treatment, $N$. benthamiana leaves were collected and RT-qPCR was used to detect the expression patterns of the 16 selected genes.

The bacterial pathogen Pst DC300 down-regulated the

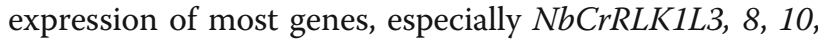
16, 18, 20, 28, and 30 (Fig. 6). TuMV infection significantly down-regulated these genes, except for NbCrRLK1L31, to 0.06-0.55. PMMoV and PVX also 


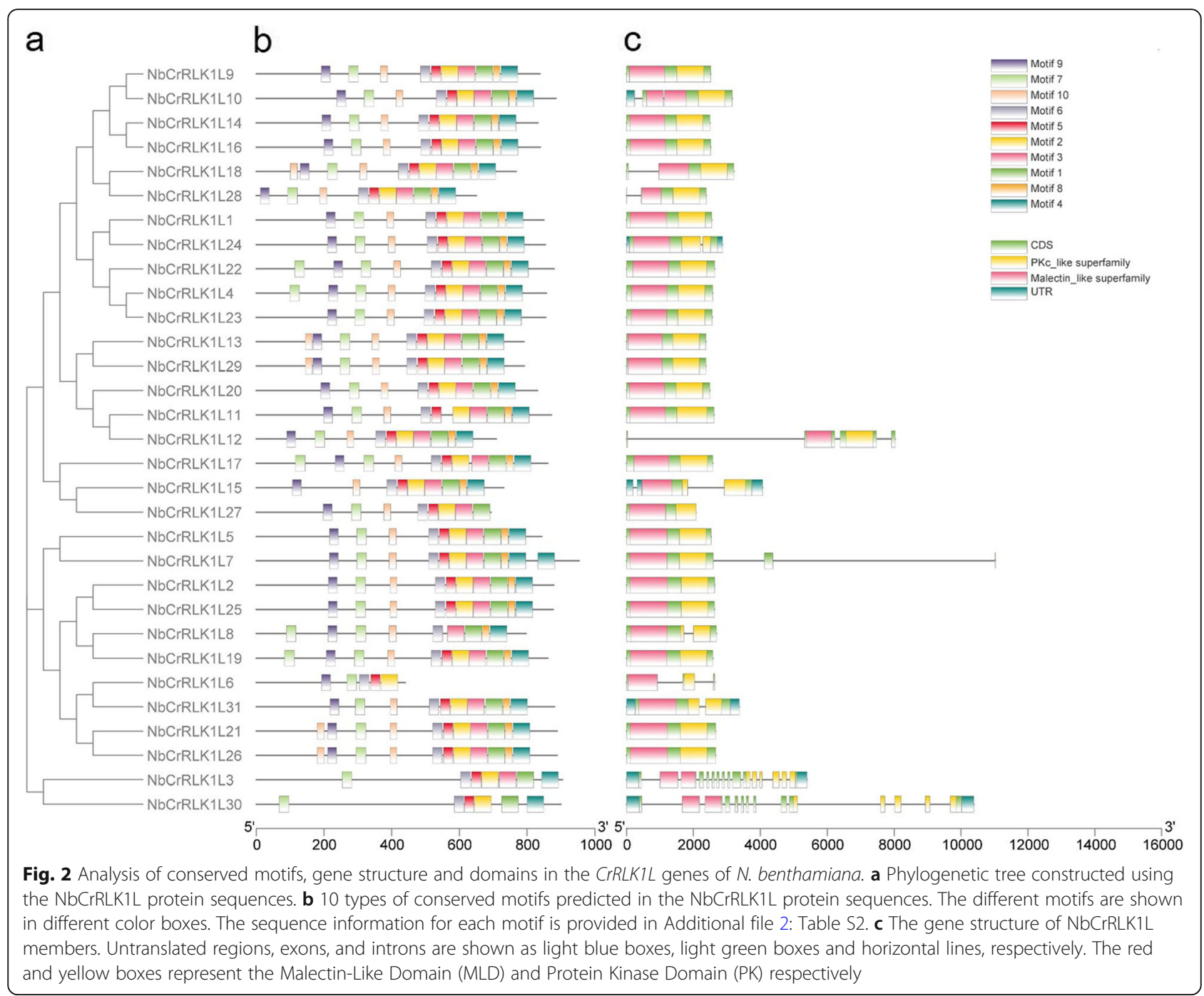

down-regulated the expression of some genes, but not to the same extent as Pst DC3000 and TuMV. Flg22 and chitin induced the expression of NbCrRLK1L3, 17, 20 and slightly down-regulated the expression of some genes, such as $N b C r R L K 1 L 8,10,18$, and 28 (Fig. 6).

\section{Discussion}

Malectin-like domains are lectin-like motifs found in eukaryotic and prokaryotic proteins. They are particularly abundant in plants and perform very important signal functions in defense and development [7, 37]. The MLD gene has been identified in the genomes of Arabidopsis, rice, cotton, poplar, and strawberry etc. $[1,32-$ 36], but this subfamily has not been identified in $N$. benthamiana. In this study a total of 31 members were identified in $N$. benthamiana. Solis-Miranda et al. identified 16 and 25 CrRLK1L members in Solanum lycopersicum and Solanum tuberosum, respectively, indicating that there were differences in the number of CrRLK1L genes in different species of the same family [36]. Based on sequence similarity, they could be divided into six groups. Five of the groups have homologous Arabidopsis gene members but the sixth group does not, suggesting that the evolution of this subfamily in different species is independent. Solis-Miranda et al. analyzed the CrRLK1L protein in 57 species and found that members of this subfamily experienced diversification in a wide range of plant groups [36]. We compared the numbers of 11 types of CrRLK1L genes in N. benthamiana, Solanum lycopersicum and Solanum tuberosum, and found that $N$. benthamiana had larger numbers of four types of homologous genes (ANXUR, HERKULES1, BUPS and THESEUS) than the Solanum species, indicating that the first, second, and fifth groups of $N b C r R L K 1 L$ may have expanded. $N b C r R L K 1 L 8 / 11 / 18 / 30$ are highly expressed in flowers, which may be related to the function of CrRLK1L subfamily during fertilization. Except for $N b C r R L K 1 L 8$, the other genes were highly expressed in 


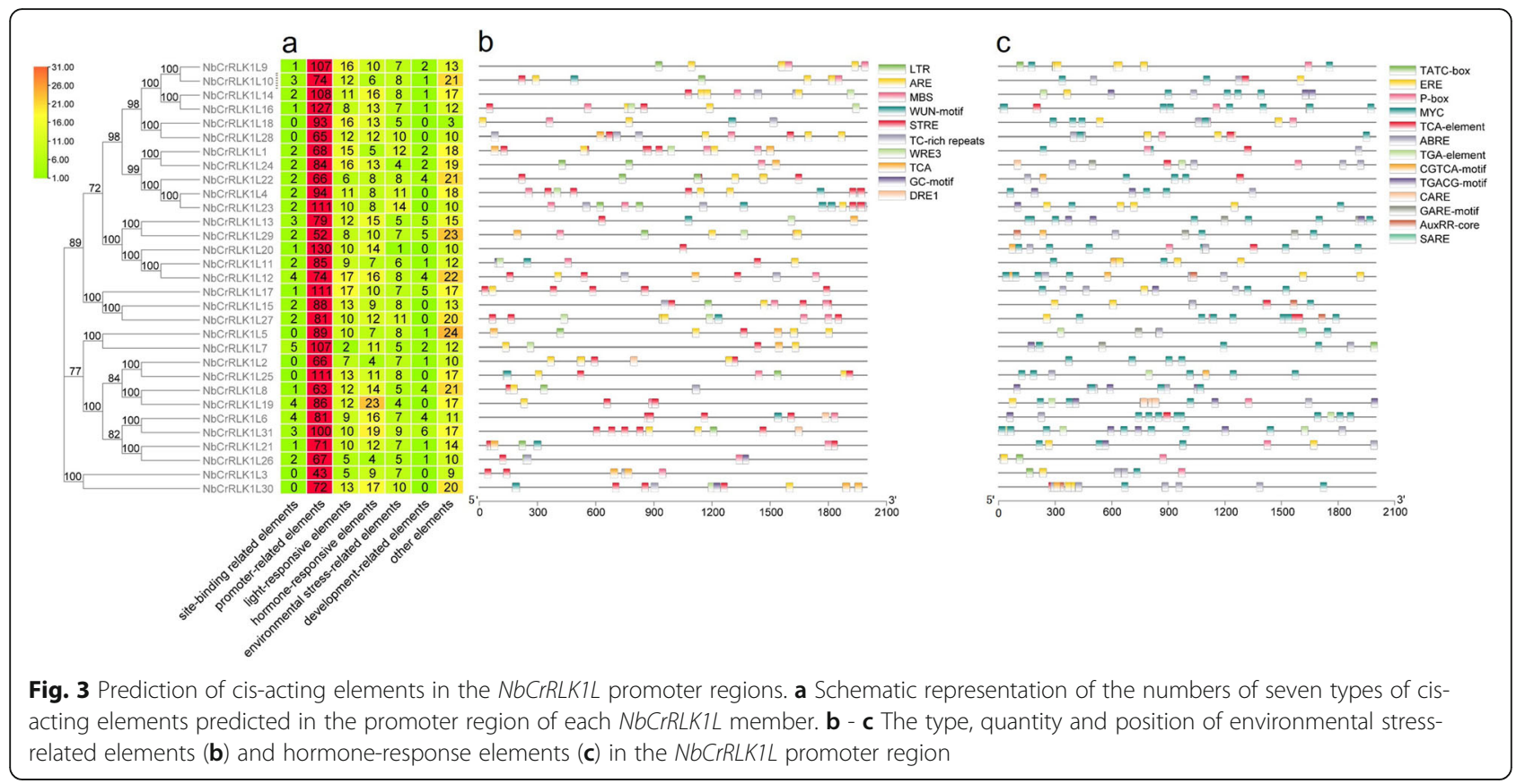

young leaves while $N b C r R L K 1 L 11 / 15 / 16 / 17 / 21 / 26 / 28 /$ 29/31 (and especially $N b C r R L K 1 L 17 / 31$ ) were also highly expressed in mature leaves, indicating the functional diversity of subfamily members. These results will provide a basis for further functional classification of NbCrRLK1Ls.

Inoculation with pathogenic bacteria (Pst DC3000) and viruses (TuMV, PMMoV, PVX) can inhibit the expression of most of the genes in the NbCrRLK1L subfamily, indicating that $\mathrm{NbCrRLK1Ls}$ can respond to pathogen infection. Lindner et al. also found that inoculation with Pst DC3000 significantly down-regulated the expression of Arabidopsis CrRLK1Ls [9]. Interestingly, in addition to inhibiting the gene expression of $\mathrm{NbCrRLK1Ls,} \mathrm{pathogens} \mathrm{can} \mathrm{also} \mathrm{induce} \mathrm{expression} \mathrm{of}$ certain genes after infection. For example, DC3000 upregulated the expression of $\mathrm{NbCrRLK1L5}$ and TuMV up-regulated NbCrRLK1L31 about 20 fold. The functions of these two genes in the process of infection by these pathogens needs to be explored further.

\section{Conclusion}

This study performs an analysis of the NbCrRLK1Ls and provides the basis for a better understand of their varied functions in plant development and in plant-microbe interactions.

\section{Methods}

Identification of NbCrRLK1L subfamily members

The protein sequences of 17 Arabidopsis CrRLK1Ls were downloaded from TAIR, and the genome of Nicotiana benthamiana was downloaded from the Sol Genomics Network (https://solgenomics.net/) [38]. NbCrRLK1Ls were identified by two rounds of BLASTP. First, all Arabidopsis CrRLK1L protein sequences were used to search for possible NbCrRLK1L sequences using TBtools [39]. Then NCBI's Batch CD-Search function was used to confirm whether the candidate NbCrRLK1Ls had a characteristic malectin-like domain (pfam12819) and kinase domain (cl21453). The candidates that did not meet these conditions were eliminated. The predicted CDS length, PI, and molecular weight of NbCrRLK1Ls were determined by ExPASy [40].

\section{Phylogenetic analysis}

The protein sequences of AtCrRLK1L and NbCrRLK1L were used to construct a phylogenetic tree using the neighbor-joining (NJ) method in Mega X software with 1000 bootstrap replicates. The tree was further annotated using iTOL [41].

\section{Analysis of conserved motifs, gene structure and functional domains}

The conserved motifs of the genes were analyzed by the MEME program [42] with the following parameters: optimal motif width was set to 30-70, the number of repetitions was set to zero or one, the maximum number of motifs was set to identify 10 motifs. Gene structure and functional domains were analyzed and visualized using NCBI Batch CD-Search and TBtools. 


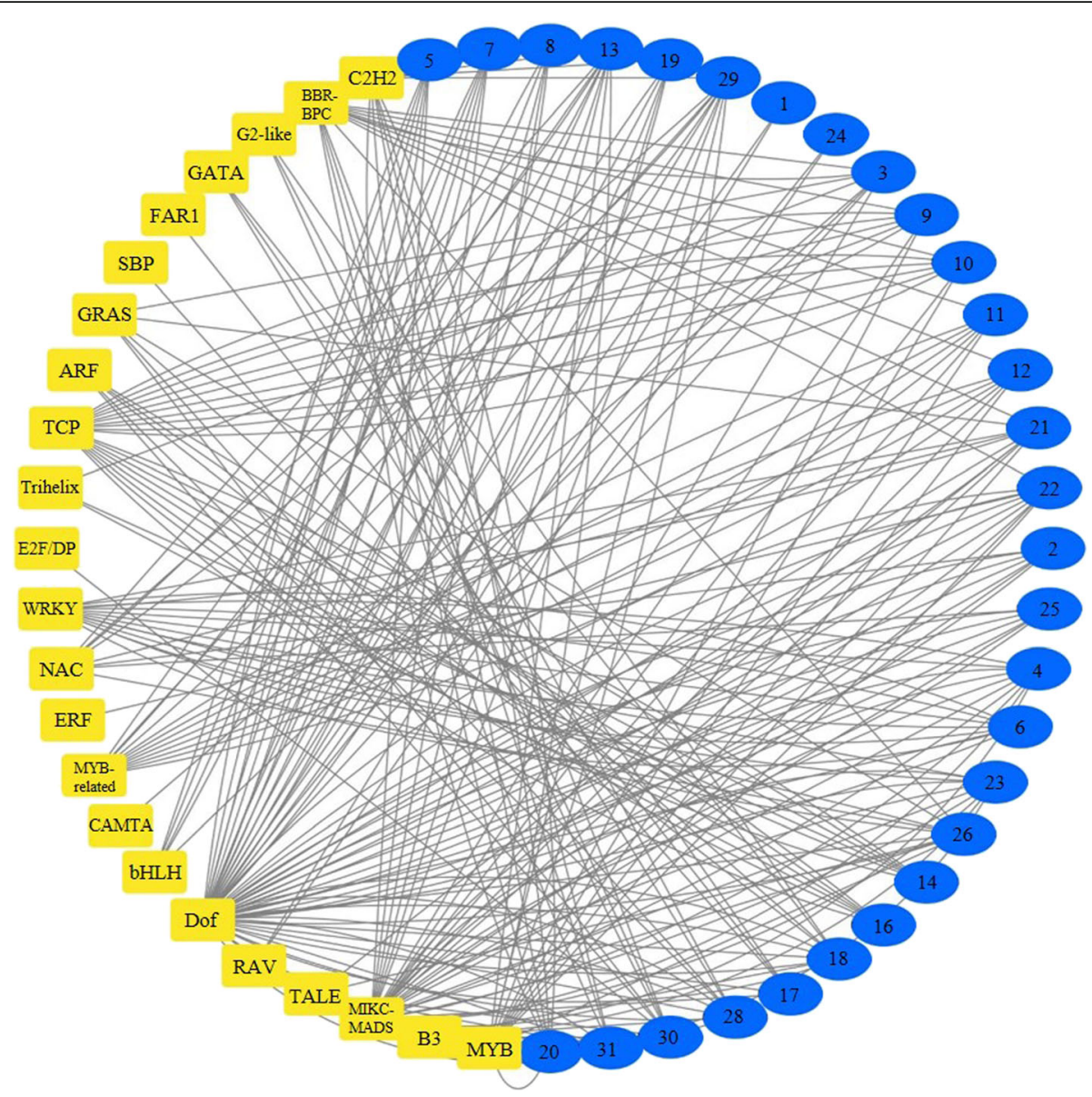

Fig. 4 Regulatory network between NbCrRLK1L and possible transcription factors. The blue ovals represent NbCrRLK1L, the yellow rectangles represent possible transcription factors, and the black lines represent possible regulatory relationships

\section{Prediction of promoter cis-acting elements and transcription factors}

Promoter cis-acting elements were predicted by PlantCARE [43] and visualized by TBtools. Transcription factors were predicted by PlantRegMap [44], and $N$. sylvestris was set as the target analysis species. Cytoscape 3.7.1 was used to visualize the target relationship between transcription factors and NbCrRLK1Ls [45].

\section{Pathogen inoculation and PAMPs treatment}

Plant growth conditions were as described previously [46]. The concentration of TuMV, PMMoV agrobacterium solution was adjusted to $\mathrm{OD}_{600}=0.1$, and the concentration of PVX agrobacterium solution was adjusted to $\mathrm{OD}_{600}=1 \times 10^{-4}$. The $N$. benthamiana leaves were injected with a needleless syringe, and the leaves injected with the transient transfection solution were used as a blank control. Pst DC3000 was cultured on King's B medium at $28{ }^{\circ} \mathrm{C}$ for 2 days. The concentration of Pst
DC3000 suspension was adjusted to $\mathrm{OD}_{600}=1 \times 10^{-5}$ to infiltrate the leaves, and the leaves injected with $10 \mathrm{mM}$ $\mathrm{MgCl}_{2}$ were used as a blank control [46]. $1 \mu \mathrm{m}$ flg22 and $200 \mu \mathrm{g} / \mathrm{mL}$ chitin dissolved in deionized water, containing $0.01 \%$ Silwet L-77, were evenly sprayed on the fully extended $N$. benthamiana leaves, and the leaves only sprayed with deionized water were used as controls. The flg22 peptide was synthesized by Sangon Biotech (Shanghai).

\section{Gene expression analysis}

Total RNA was extracted by the TRIZOL method, and $1 \mu \mathrm{g}$ total RNA was used for reverse transcription using the Toyobo cDNA First Strand Synthesis Kit. RT-qPCR was then performed on a Roche LightCycler ${ }^{\circ} 480$ RealTime PCR instrument with Toyobo Premix Kit. Three independent biological replicates with three technical replicates were performed. All primers are listed in the Additional file 6: Table S5. 


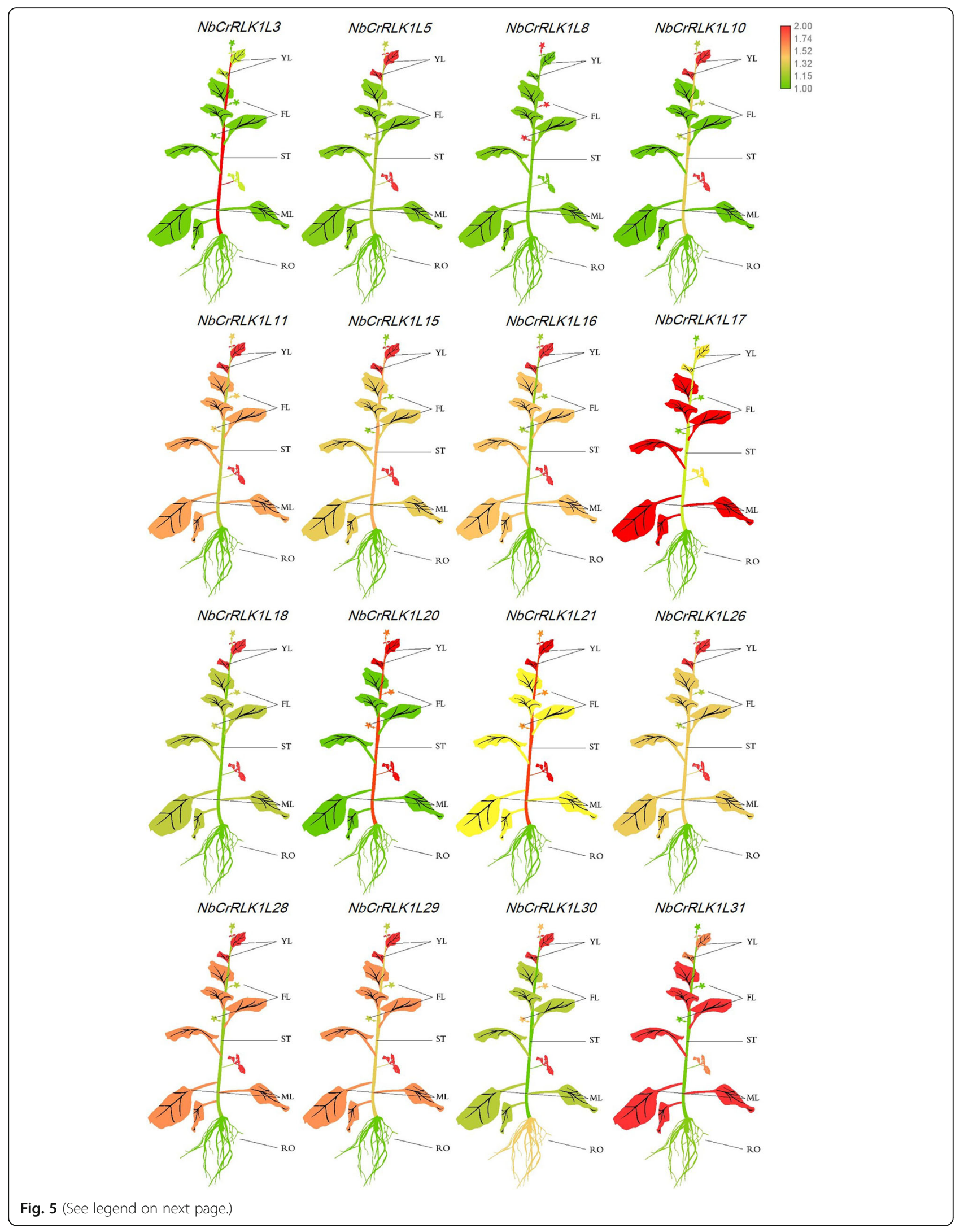


(See figure on previous page.)

Fig. 5 Expression levels of representative NbCrRLK1Ls in different tissues. The mean expression value was calculated from three independent biological replicates relative to that in young leaves. YL: young leaf; ML: mature leaf; ST: stem; RO: root; FL: flower. Red represents a high expression level and green represents a low expression level. The raw data of relative expression values and standard errors is provided in Additional file 5: Figure S1

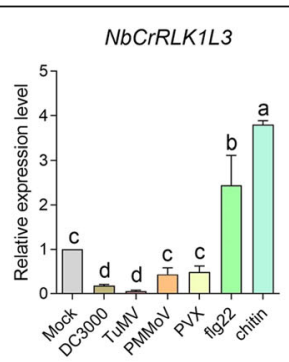

NbCrRLK1L11

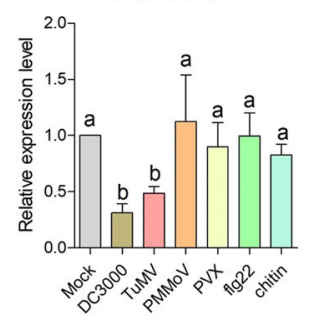

NbCrRLK1L18

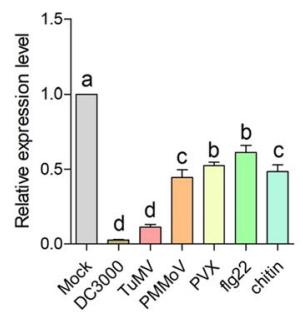

NbCrRLK1L28

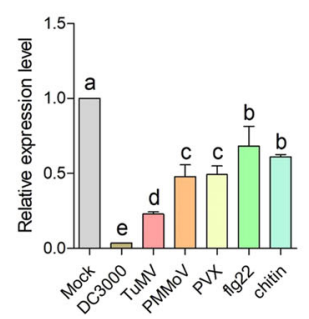

NbCrRLK1L5

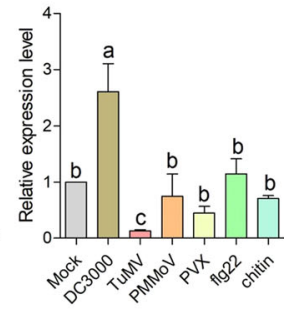

NbCrRLK1L15

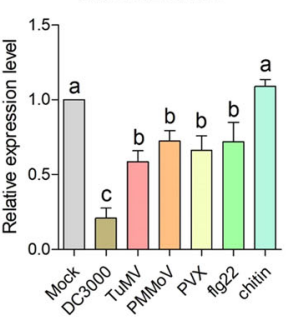

NbCrRLK1L2O

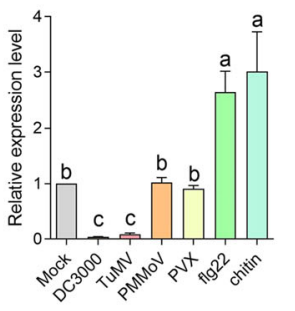

NbCrRLK1L29

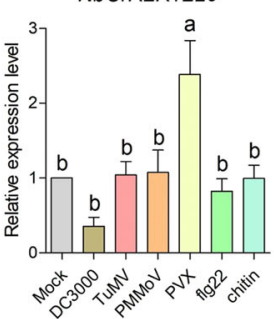

NbCrRLK1L8

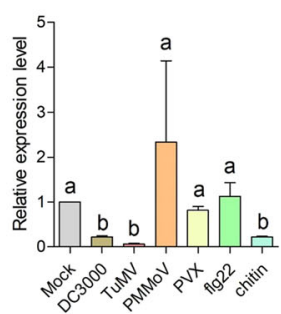

NbCrRLK1L16

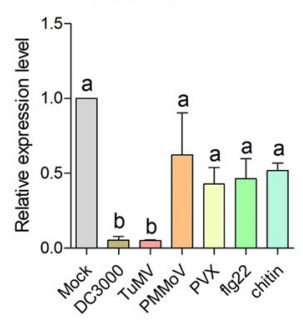

NbCrRLK1L21

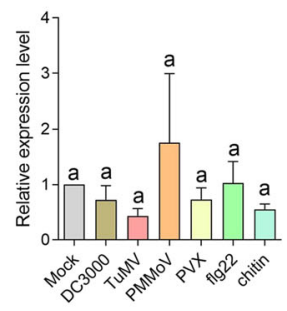

NbCrRLK1L30

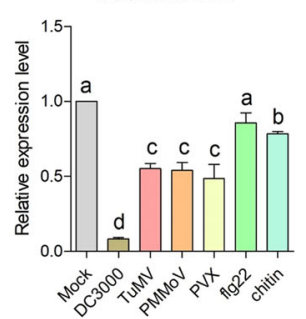

NbCrRLK1L10

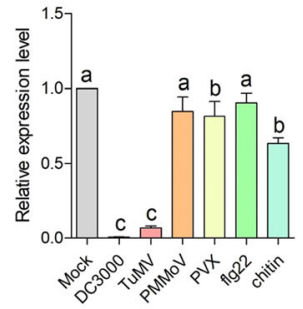

NbCrRLK1L17

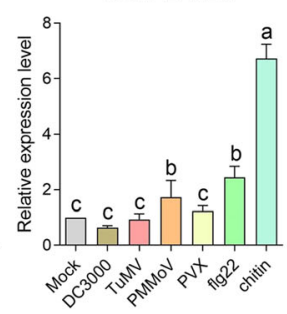

NbCrRLK1L26

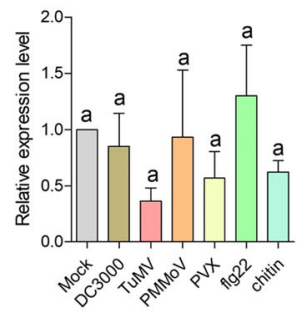

NbCrRLK1L31

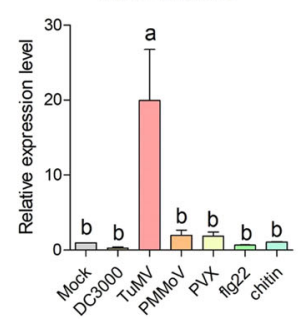

Fig. 6 Expression analysis of representative NbCrRLK1Ls after treatment of $N$. benthamiana with different pathogens or PAMPs. The mean expression values were calculated from three independent biological replicates and were relative to mock-inoculated controls 


\section{Supplementary Information}

The online version contains supplementary material available at https://doi. org/10.1186/s12870-021-03208-x.

Additional file 1: Table S1. List of NbCrRLK1L protein sequences. Additional file 2: Table S2. The MEME motif sequences and lengths of NbCrRLK1LS.

Additional file 3: Table S3. Cis-acting elements in NbCrRLK1LS. Additional file 4: Table S4. Potential transcription factors of NbCrRLK1LS.

Additional file 5: Figure S1. Expression levels of representative NbCrRLK1Ls in different tissues (raw data).

Additional file 6: Table S5. Primers used in this study.

\section{Acknowledgements}

The authors would like to thank Dr. Yule Liu (Tsinghua University, China) for kindly providing Pst DC3000, Dr. Fernando Ponz (Universidad Politécnica de Madrid, Spain) and Dr. Stuart MacFarlane (James Hutton Institute, UK) for sharing TuMV and PVX infectious clones and Prof. M. J. Adams (Minehead, UK) for critical reading of the manuscript.

\section{IUCN policy statement}

Our experimental research on $\mathrm{N}$. benthamiana, including the collection of plant material, complied with relevant institutional, national, and international guidelines and legislation.

\section{Authors' contributions}

S. R. and F. Y. designed the experiments, S. R., X. W., H. Z., Y. L., J. P. and G. W. performed the experiments and collected the data. S. R., X. W. and F. Y. analyzed the data. S. R. wrote the manuscript. F. Y. and J. C. revised the manuscript. All authors have read and approved the manuscript.

\section{Funding}

This work was financially supported by the Chinese Agriculture Research System (CARS-24-C-04) and K. C. Wong Magna Fund in Ningbo University. The funders did not participate in the design of this study, data collection, analysis, and manuscript writing.

\section{Availability of data and materials}

All data generated or analyzed during this study are included in this published article and its additional files. The datasets used and analyzed during the current study are available from the corresponding author on reasonable request. The analysis websites used in this study are as follows: Batch CD-Search (https://www.ncbi.nlm.nih.gov/Structure/bwrpsb/bwrpsb. cgi), ExPASy (https://www.expasy.org/), iTOL (https://itol.embl.de/index.shtml), MEME (http://meme-suite.org/tools/meme), PlantCARE (http://bioinformatics. psb.ugent.be/webtools/plantcare/htm//), PlantRegMap (http://plantregmap. gao-lab.org/network.php). The public's access to these databases is open and there is no sequencing data in this study.

\section{Declarations}

Ethics approval and consent to participate Not applicable.

\section{Consent for publication}

Not applicable.

\section{Competing interests}

The authors declare no conflicts of interest.

\section{Author details}

${ }^{1}$ State Key Laboratory for Managing Biotic and Chemical Threats to the Quality and Safety of Agro-products, Key Laboratory of Biotechnology in Plant Protection of Ministry of Agriculture and Zhejiang Province, Institute of Plant Virology, Ningbo University, Ningbo 315211, China. ${ }^{2}$ College of Life Science, China Jiliang University, Hangzhou 310058, China.
Received: 8 May 2021 Accepted: 1 September 2021 Published online: 18 September 2021

\section{References}

1. Shiu SH, Karlowski WM, Pan R, Tzeng YH, Mayer KF, et al. Comparative analysis of the receptor-like kinase family in Arabidopsis and rice. Plant Cell. 2004;16(5):1220-34. https://doi.org/10.1105/tpc.020834.

2. Shiu SH, Bleecker AB. Expansion of the receptor-like kinase/Pelle gene family and receptor-like proteins in Arabidopsis. Plant Physiol. 2003;132(2):530-43. https://doi.org/10.1104/pp.103.021964.

3. He Y, Zhou J, Shan L, Meng X. Plant cell surface receptor-mediated signaling - a common theme amid diversity. J Cell Sci. 2018;131:jcs209353.

4. Gish LA, Clark SE. The RLK/Pelle family of kinases. Plant J. 2011;66(1):117-27. https://doi.org/10.1111/j.1365-313X.2011.04518.X.

5. Smakowska-Luzan E, Mott GA, Parys K, Stegmann M, Howton TC, Layeghifard $M$, et al. An extracellular network of Arabidopsis leucine-rich repeat receptor kinases. Nature. 2018;553(7688):342-6. https://doi.org/10.1038/nature25184.

6. Breiden M, Simon R. Q\&A: How does peptide signaling direct plant development? BMC Biol. 2016;14(1):58. https://doi.org/10.1186/s12915-0160280-3.

7. Franck CM, Westermann J, Boisson-Dernier. A plant malectin-like receptor kinases: from cell wall integrity to immunity and beyond. Annu Rev Plant Biol. 2018;69(1):301-28. https://doi.org/10.1146/annurev-arplant-042817-040557.

8. Galindo-Trigo S, Gray JE, Smith LM. Conserved roles of CrRLK1L receptor-like kinases in cell expansion and reproduction from algae to angiosperms. Front Plant Sci. 2016:7:1269. https://doi.org/10.3389/fpls.2016.01269.

9. Lindner H, Muller LM, Boisson-Dernier A, Grossniklaus U. CrRLK1L receptorlike kinases: not just another brick in the wall. Curr Opin Plant Biol. 2012; 15(6):659-69. https://doi.org/10.1016/j.pbi.2012.07.003.

10. Escobar-Restrepo JM, Huck N, Kessler S, Gagliardini V, Gheyselinck J, Yang WC, et al. The FERONIA receptor-like kinase mediates male-female interactions during pollen tube reception. Science. 2007;317(5838):656-60. https://doi.org/10.1126/science.1143562.

11. Duan Q, Kita D, Li C, Cheung AY, Wu HM. FERONIA receptor-like kinase regulates $\mathrm{RHO}$ GTPase signaling of root hair development. Proc Natl Acad Sci U S A. 2010;107(41):17821-6. https://doi.org/10.1073/pnas.1005366107.

12. Li C, Yeh FL, Cheung AY, Duan Q, Kita D, Liu MC, et al. Glycosylphosphatidylinositol-anchored proteins as chaperones and coreceptors for FERONIA receptor kinase signaling in Arabidopsis. Elife. 2015;4: e06587. https://doi.org/10.7554/eLife.06587.

13. Boisson-Dernier A, Roy S, Kritsas K, Grobei MA, Jaciubek M, et al. Disruption of the pollen-expressed FERONIA homologs ANXUR1 and ANXUR2 triggers pollen tube discharge. Development. 2009;136(19):3279-88. https://doi.org/1 0.1242/dev.040071.

14. Miyazaki S, Murata T, Sakurai-Ozato N, Kubo M, Demura T, Fukuda H, et al. ANXUR1 and 2, sister genes to FERONIAVSIRENE, are male factors for coordinated fertilization. Curr Biol. 2009;19(15):1327-31. https://doi.org/10.1 016/j.cub.2009.06.064.

15. Ge Z, Bergonci T, Zhao Y, Zou Y, Du S, Liu MC, et al. Arabidopsis pollen tube integrity and sperm release are regulated by RALF-mediated signaling. Science. 2017;358(6370):1596-600. https://doi.org/10.1126/science.aao3642.

16. Hematy K, Sado PE, Van Tuinen A, Rochange S, Desnos T, et al. A receptor-like kinase mediates the response of Arabidopsis cells to the inhibition of cellulose synthesis. Curr Biol. 2007;17(11):922-31. https://doi.org/10.1016/j.cub.2007.05.018.

17. Bai L, Ma X, Zhang G, Song S, Zhou Y, Gao L, et al. A receptor-like kinase mediates ammonium homeostasis and is important for the polar growth of root hairs in Arabidopsis. Plant Cell. 2014;26(4):1497-511. https://doi.org/1 $0.1105 /$ tpc. 114.124586

18. Gachomo EW, Baptiste LJ, Kefela T, et al. The Arabidopsis CURVY1 (CVY1) gene encoding a novel receptor-like protein kinase regulates cell morphogenesis, flowering time and seed production. BMC Plant Biol. 2014; 14(1):221. https://doi.org/10.1186/s12870-014-0221-7.

19. Schoenaers S, Balcerowicz D, Costa A, Vissenberg K. The kinase ERULUS controls pollen tube targeting and growth in Arabidopsis thaliana. Front Plant Sci. 2017;8:1942. https://doi.org/10.3389/fpls.2017.01942.

20. Richter J, Watson JM, Stasnik P, Borowska M, Neuhold J, Berger M, et al. Multiplex mutagenesis of four clustered CrRLK1L with CRISPR/Cas9 exposes their growth regulatory roles in response to metal ions. Sci Rep. 2018;8(1): 12182. https://doi.org/10.1038/s41598-018-30711-3.

21. Stegmann M, Monaghan J, Smakowska-Luzan E, Rovenich H, Lehner A, Holton N, et al. The receptor kinase FER is a RALF-regulated scaffold 
controlling plant immune signaling. Science. 2017;355(6322):287-9. https:// doi.org/10.1126/science.aal2541.

22. Haruta M, Sabat G, Stecker K, Minkoff BB, Sussman MR. A peptide hormone and its receptor protein kinase regulate plant cell expansion. Science. 2014; 343(6169):408-11. https://doi.org/10.1126/science.1244454.

23. Wu J, Kurten EL, Monshausen G, Hummel GM, Gilroy S, Baldwin IT. NaRALF, a peptide signal essential for the regulation of root hair tip apoplastic $\mathrm{pH}$ in Nicotiana attenuata, is required for root hair development and plant growth in native soils. Plant J. 2007;52(5):877-90. https://doi.org/10.1111/j.1365-313 X.2007.03289.X.

24. Covey PA, Subbaiah CC, Parsons RL, Pearce G, Lay FT, Anderson MA, et al. A pollen-specific RALF from tomato that regulates pollen tube elongation. Plant Physiol. 2010;153(2):703-15. https://doi.org/10.1104/pp.110.155457.

25. Campbell L, Turner SR. A comprehensive analysis of RALF proteins in green plants suggests there are two distinct functional groups. Front Plant Sci. 2017:8:37. https://doi.org/10.3389/fpls.2017.00037.

26. do Canto AM, Ceciliato PH, Ribeiro B, Ortiz Morea FA, Franco Garcia AA, et al. Biological activity of nine recombinant AtRALF peptides: implications for their perception and function in Arabidopsis. Plant Physiol Biochem. 2014;75:45-54. https://doi.org/10.1016/j.plaphy.2013.12.005.

27. Bergonci T, Ribeiro B, Ceciliato PH, Guerrero-Abad JC, Silva-Filho MC, et al. Arabidopsis thaliana RALF1 opposes brassinosteroid effects on root cell elongation and lateral root formation. J Exp Bot. 2014;65(8):2219-30. https:// doi.org/10.1093/jxb/eru099.

28. Xiao Y, Stegmann M, Han Z, DeFalco TA, Parys K, et al. Mechanisms of RALF peptide perception by a heterotypic receptor complex. Nature. 2019; 572(7768):270-4. https://doi.org/10.1038/s41586-019-1409-7.

29. Guo H, Nolan TM, Song G, Liu S, Xie Z, Chen J, et al. FERONIA receptor kinase contributes to plant immunity by suppressing jasmonic acid signaling in Arabidopsis thaliana. Curr Biol. 2018;28(20):3316-24. https://doi. org/10.1016/j.cub.2018.07.078.

30. Mang H, Feng B, Hu Z, Boisson-Dernier A, Franck CM, Meng X, et al. Differential regulation of two-tiered plant immunity and sexual reproduction by ANXUR receptor-like kinases. Plant Cell. 2017;29(12):314056. https://doi.org/10.1105/tpc.17.00464

31. Liu J, Huang Y, Kong L, Yu X, Feng B, Liu D, et al. The malectin-like receptor-like kinase LETUM1 modulates NLR protein SUMM2 activation via MEKK2 scaffolding. Nat Plants. 2020;6(9):1 106-15. https://doi.org/10.1038/s41477-020-0748-6.

32. Nguyen QN, Lee YS, Cho LH, Jeong HJ, An G, Jung KH. Genome-wide identification and analysis of Catharanthus roseus RLK1-like kinases in rice. Planta. 2015;241(3):603-13. https://doi.org/10.1007/s00425-014-2203-2.

33. Niu E, Cai C, Zheng Y, Shang X, Fang L, Guo W. Genome-wide analysis of CrRLK1L gene family in Gossypium and identification of candidate CrRLK1L genes related to fiber development. Mol Gen Genomics. 2016;291(3):113754. https://doi.org/10.1007/s00438-016-1169-0.

34. Kumar V, Donev EN, Barbut FR, Kushwah S, Mannapperuma C, Urbancsok J, et al. Genome-wide identification of Populus malectin/malectin-like domaincontaining proteins and expression analyses reveal novel candidates for signaling and regulation of wood development. Front Plant Sci. 2020;11 588846. https://doi.org/10.3389/fpls.2020.588846.

35. Zhang Q, Jia M, Xing Y, Qin L, Li B, Jia W. Genome-wide identification and expression analysis of MRLK family genes associated with strawberry (Fragaria vesca) fruit ripening and abiotic stress responses. PLoS One. 2016; 11(9):e0163647. https://doi.org/10.1371/journal.pone.0163647.

36. Solis-Miranda J, Fonseca-Garcia C, Nava N, Pacheco R, Quinto C. Genomewide identification of the CrRLK1L subfamily and comparative analysis of its role in the legume-rhizobia symbiosis. Genes (Basel). 2020;11:793.

37. Bellande K, Bono JJ, Savelli B, Jamet E, Canut H. Plant lectins and lectin receptor-like kinases: how do they sense the outside? Int J Mol Sci. 2017; 18(6):1164. https://doi.org/10.3390/ijms18061164.

38. Bombarely A, Rosli HG, Vrebalov J, Moffett P, Mueller LA, Martin GB. A draft genome sequence of Nicotiana benthamiana to enhance molecular plantmicrobe biology research. Mol Plant-Microbe Interact. 2012;25(12):1523-30. https://doi.org/10.1094/MPMI-06-12-0148-TA.

39. Chen C, Chen H, Zhang Y, Thomas HR, Frank MH, He Y, et al. TBtools: An integrative toolkit developed for interactive analyses of big biological data. Mol Plant. 2020;13(8):1194-202. https://doi.org/10.1016/j.molp.2020.06.009.

40. Wilkins MR, Gasteiger EG, Bairoch A, Sanchez JC, Williams KL, Appel RD, et al. Protein identification and analysis tools in the ExPASy server. Methods Mol Biol. 1999;112:531-52. https://doi.org/10.1385/1-59259-584-7:531.
41. Letunic I, Bork P. Interactive Tree Of Life (iTOL): an online tool for phylogenetic tree display and annotation. Bioinformatics. 2007;23(1):127-8. https://doi.org/10.1093/bioinformatics/btl529.

42. Bailey TL, Boden M, Buske FA, Frith M, Grant CE, Clementi L, et al. MEME Suite: tools for motif discovery and searching. Nucleic Acids Res. 2009; 37(Web Server):W202-8. https://doi.org/10.1093/nar/gkp335.

43. Lescot M, Déhais $P$, Thijs $G$, Marchal $K$, Moreau $Y$, van de Peer $Y$, et al. PlantCARE, a database of plant cis-acting regulatory elements and a portal to tools for in silico analysis of promoter sequences. Nucleic Acids Res. 2002;30(1):325-7. https://doi.org/10.1093/nar/30.1.325.

44. Jin J, Tian F, Yang DC, Meng YQ, Kong L, Luo J, et al. PlantTFDB 4.0: toward a central hub for transcription factors and regulatory interactions in plants. Nucleic Acids Res. 2017;45(D1):D1040-5. https://doi.org/10.1093/nar/gkw982.

45. Su G, Morris JH, Demchak B, Bader GD. Biological network exploration with Cytoscape 3. Curr Protoc Bioinformatics. 2014;47(1):8131-24. https://doi. org/10.1002/0471250953.bi0813s47.

46. Wu X, Lai Y, Lv L, Ji M, Han K, Yan D, et al. Fasciclin-like arabinogalactan gene family in Nicotiana benthamiana: genome-wide identification, classification and expression in response to pathogens. BMC Plant Biol. 2020;20(1):305. https://doi.org/10.1186/s12870-020-02501-5.

\section{Publisher's Note}

Springer Nature remains neutral with regard to jurisdictional claims in published maps and institutional affiliations.
Ready to submit your research? Choose BMC and benefit from:

- fast, convenient online submission

- thorough peer review by experienced researchers in your field

- rapid publication on acceptance

- support for research data, including large and complex data types

- gold Open Access which fosters wider collaboration and increased citations

- maximum visibility for your research: over $100 \mathrm{M}$ website views per year

At BMC, research is always in progress.

Learn more biomedcentral.com/submissions 